\title{
$X(3872)$ and its charmonium content in Heavy Quark limit
}

\author{
Elif Cincioglu ${ }^{1}$, Juan Nieves ${ }^{2}$, and Altug Ozpineci ${ }^{3, \star}$ \\ ${ }^{1}$ Physics Engineering, Ankara University, Ankara, Turkey \\ ${ }^{2}$ IFIC, Centro Mixto Universidad de Valencia-CSIC, Valencia, Spain \\ ${ }^{3}$ Physics Department, Middle East Technical University, Ankara, Turkey
}

\begin{abstract}
X(3872)$ still presents many puzzles more than a decade after its discovery. Some of its properties, like the isospin violating decays, can easily be accommodated in a molecular model, whereas its other properties, such as radiative decays can be more naturally explained in the quarkonium picture. The best of these schemes can be combined in a picture of $X(3872)$ where it is dominantly a molecular state with some charmonium components. In this work, we present a model based on heavy quark symmetry which describes $X(3872)$ as a superposition of molecular and charmonium components.
\end{abstract}

\section{Introduction}

The hadronic spectroscopy has witnessed an exciting period in the recent years. Some of this excitement is due to the observation of mesons that can not be easily explained using the conventional quark-anti-quark picture. One of these mesons is the $X(3872)$ meson which was first observed more than a decade ago by the Belle collaboration [1]. Its existence was later on confirmed by other experiments [2-5]. Its quantum numbers have been confirmed to be $J^{P C}=1^{++}$only recently by the $\mathrm{LHCb}$ experiment [5]. To explain its properties, various models have been proposed, some of the most common ones are the charm-anti-charm, molecular and tetraquark pictures. In this work, we present a consistent framework based on heavy quark symmetry which tries to combine the pure charmonium and the molecular pictures. Details of the model and its analysis can be found in [6].

In a constituent quark model description of $X(3872)$ it can be identified by the $2 P$ state. Various predictions for the mass of the $2 P$ state range from $3947.4 \mathrm{MeV}$ [7, 8], to $3906 \mathrm{MeV}$ [9]. All of the predictions on the mass of the $2 P$ state are at least $30 \mathrm{MeV}$ above the observed mass of $X(3872)$, which is just below the $D^{0} D^{* 0}$ threshold. This "coincidence" can easily be explained using a molecular picture for the $X(3872)$. In the molecular picture, the mass of the $X(3872)$ is $m_{X}=m_{D^{0}}+m_{D^{* 0}}-B$, where $B>0$ is the binding energy, i.e. in the molecular picture $X(3872)$ is a weakly bound $D \bar{D}^{*}$ state.

Another puzzling observation about $X(3872)$ is its decays into final states containing a $J / \psi$ and two or three pions. These decays proceed through intermediate $\rho$ and $\omega$ mesons. Taking into account the phase space difference, the ratio of the decay amplitudes are obtained to be [10]

$$
\left|\frac{A(J / \psi \rho)}{A(J / \psi \omega)}\right|=0.27 \pm 0.02
$$

^e-mail: ozpineci@metu.edu.tr 
Considering that $J / \psi \rho$ is an $I=1$ and $J / \psi \omega$ is an $I=0$ final state, this ratio points out to a large isospin violation. Such a large isospin violation is hard to reconcile with a pure charmonium state where $X(3872)$ would have $I=0$ and the observed violation should arise during the decay. In contrast, in the molecular picture, due to the mass difference between the neutral and charged $D$ mesons, $X(3872)$ is a mixture of $I=0$ and $I=1$ states. In such a picture, the mixing angle between the $I=0$ and $I=1$ components have been estimated to be almost maximal $\theta_{\text {mixing }} \simeq 40^{\circ}[11,12]$. Hence the observed isospin violation can easily arise due to this mixing, and not due to the processes that lead to the decay.

When radiative decays of $X(3872)$ are considered, quark-anti-quark picture of $X(3872)$ is favored. $X(3872)$ is observed to decay into $\psi(2 S)$ and $\psi(1 S)$ by the emission of one photon. The ratio of the branching ratios is measured to be $[13,14]$

$$
R_{\psi \gamma}=\frac{B r(X \rightarrow \psi(2 S) \gamma)}{B r(X \rightarrow J / \psi \gamma)}=2.46 \pm 0.64 \pm 0.29
$$

This ratio can be accommodated into the molecular picture if one assumes that $D D^{*} \psi(2 S)$ coupling is about twice as large as $D D^{*} J / \psi$ coupling. In the charm-anti-charm picture, $R_{\psi \gamma}$ can be explained more naturally since the $X \rightarrow \psi(2 S) \gamma$ transition is nothing but the $\Delta l=1$ transition.

Finally, it is observed that in $p p$ collisions, the production rate of $X(3872)$ is $1 / 20$ times the production rate of $\psi(2 S)$ [15]. Such a large rate can not be naturally explained in the pure molecular picture. This problem can be avoided if one assumes that $X(3872)$ is dominantly a molecular state with about $5 \%$ charmonium admixture.

In the next section, a leading order effective Lagrangian consistent with the heavy quark symmetry will be presented where $X(3872)$ arises as a mixture of a molecule and charmonium. Section III will be devoted to the $J^{P C}=1^{++}$and $J^{P C}=2^{++}$spectrum of the model followed by our conclusions.

\section{Leading order effective Lagrangian}

When the heavy quark mass is taken to be infinite, $m_{Q} \rightarrow \infty$, a new symmetry, the so called heavy quark symmetry, arises due to the decoupling of the spin of the heavy quark from the dynamics. In this limit, $D$ and $D^{*}$ mesons can be grouped into a doublet field as:

$$
\begin{aligned}
H_{a}^{(Q)} & =\frac{1+\not p}{2}\left(P_{a \mu}^{*(Q)} \gamma^{\mu}-P_{a}^{(Q)} \gamma_{5}\right), & v \cdot P_{a}^{*(Q)}=0, \\
H^{(\bar{Q}) a} & =\left(P_{\mu}^{*(\bar{Q}) a} \gamma^{\mu}-P^{(\bar{Q}) a} \gamma_{5}\right) \frac{1-\not b}{2}, & v \cdot P^{*(\bar{Q}) a}=0 .
\end{aligned}
$$

where $v=\lim _{m_{Q} \rightarrow \infty} \frac{p}{m_{Q}}$ is the heavy quark velocity, $a$ is the isospin index, $P^{*(Q)}$ and $P^{(Q)}$ fields annihilate vector and pseudoscalar mesons containing a heavy quark, and similarly for the fields $P^{*(\bar{Q})}$ and $P^{(\bar{Q})}$. Once the HQSS multiplets are identified, the leading order (LO) heavy quark spin symmetric interactions between the $D$ and $D^{*}$ mesons can be written as

$$
\begin{aligned}
\mathcal{L}_{4 H} & =C_{A} \operatorname{Tr}\left[\bar{H}^{(Q) a} H_{a}^{(Q)} \gamma_{\mu}\right] \operatorname{Tr}\left[H^{(\bar{Q}) a} \bar{H}_{a}^{(\bar{Q})} \gamma^{\mu}\right] \\
& +C_{A}^{\tau} \operatorname{Tr}\left[\bar{H}^{(Q) a} \vec{\tau}^{b}{ }_{\cdot a} H_{b}^{(Q)} \gamma_{\mu}\right] \operatorname{Tr}\left[H^{(\bar{Q}) c} \vec{\tau}^{d}{ }_{. c} \bar{H}_{d}^{(\bar{Q})} \gamma^{\mu}\right] \\
& +C_{B} \operatorname{Tr}\left[\bar{H}^{(Q) a} H_{a}^{(Q)} \gamma_{\mu} \gamma_{5}\right] \operatorname{Tr}\left[H^{(\bar{Q}) a} \bar{H}_{a}^{(\bar{Q})} \gamma^{\mu} \gamma_{5}\right] \\
& +C_{B}^{\tau} \operatorname{Tr}\left[\bar{H}^{(Q) a} \vec{\tau}^{b}{ }_{. a} H_{b}^{(Q)} \gamma_{\mu} \gamma_{5}\right] \operatorname{Tr}\left[H^{(\bar{Q}) c} \vec{\tau}^{d}{ }_{. c} \bar{H}_{d}^{(\bar{Q})} \gamma^{\mu} \gamma_{5}\right]
\end{aligned}
$$

This model predicts that $X(3872)$ should have heavy quark spin symmetric partners with quantum numbers $J^{P C}=0^{++}, J^{P C}=2^{++}$(denoted by $X_{2}$ in this work) and $J^{P C}=1^{+-}$whose binding energy should be similar to the binding energy of $X(3872)$ [16]. 
To include the charmonium states into this Lagrangian, their multiplet is required. The P-wave heavy quarkonia can be written as a multiplet by means of the four-vector field [17] $\left(\epsilon_{0123}=+1\right)$ :

$$
J^{\mu}=\frac{1+\not p}{2}\left(\chi_{2}^{\mu \alpha} \gamma_{\alpha}+\frac{i}{\sqrt{2}} \epsilon^{\mu \alpha \beta \gamma} \chi_{1 \gamma} v_{\alpha} \gamma_{\beta}+\frac{1}{\sqrt{3}} \chi_{0}\left(\gamma^{\mu}-v^{\mu}\right)+h^{\mu} \gamma_{5}\right) \frac{1-\not p}{2}
$$

with $J_{\mu} v^{\mu}=0$. Here, $\chi_{2}^{\mu \alpha}, \chi_{1}^{\mu}, \chi_{0}$ and $h^{\mu}$ fields annihilate $\chi_{Q J}(n P)$ and $h_{Q}(n P)$ quarkonium states, with $J^{P C}=0^{++}, 1^{++}, 2^{++}$and $1^{+-}$, respectively.

Once the multiplet is identified, there is a single heavy quark spin symmetric interaction term between the quarkonium field $J_{\mu}$ and the $H^{(Q)}, H^{(\bar{Q})}$ fields [18],

$$
\mathcal{L}_{H H Q \bar{Q}}=\frac{d}{2} \operatorname{Tr}\left[H^{a(\bar{Q})} \bar{J}_{\mu} H_{a}^{(Q)} \gamma^{\mu}\right]+\frac{d}{2} \operatorname{Tr}\left[\bar{H}^{a(Q)} J_{\mu} \bar{H}_{a}^{(\bar{Q})} \gamma^{\mu}\right]
$$

Note that in the constituent quark model, there is an infinite tower of P-wave quarkonia, all of which can couple to $D$ and $D^{*}$ mesons. Hence strictly speaking, for each radial excitation, there is such an interaction term in the Lagrangian. But assuming that the constant $d$ has the same order of magnitude for all of them, the P-wave charmonia that will mix dominantly will be the $n=2$ charmonia due to the closeness of its mass to the threshold.

Once the model is fixed, the spectrum can be identified using the poles of the $T$ matrix in each one of the $J^{P C}$ sectors. The coupling of the states to various channels can be extracted from the residues of the $T$ matrix elements near the threshold through

$$
T_{i j} \stackrel{E \rightarrow E_{R}}{\longrightarrow} \frac{g_{i} g_{j}}{E-E_{R}}
$$

where $E_{R}=M_{R}-i \frac{\Gamma_{R}}{2}$ is the position of the pole, $M_{R}$ the mass of the resonance and $\Gamma_{R}$ its width.

Identifying the nature of a resonance from the $T$ matrix elements has its complications. We will define the weights of the molecular component $(\tilde{X})$ and charmonium component $(\tilde{Y})$ through

$$
\tilde{X}=-\frac{\Sigma_{c \bar{c}}^{\prime}\left(E_{R}\right)}{1-\Sigma_{c \bar{c}}^{\prime}\left(E_{R}\right)}, \quad \tilde{Z}=\frac{1}{1-\Sigma_{c \bar{c}}^{\prime}\left(E_{R}\right)}
$$

where $\Sigma_{c \bar{c}}^{\prime}\left(E_{R}\right)$ is the derivative with respect to the energy of the self energy of the bare charmonium state due to its coupling to $D$ mesons. Strictly speaking, these parameters correspond to probabilities for bound states, whereas for resonances, their interpretation is more problematic. Nevertheless, even for resonances, they can be used as an indication of the weight of various components in the identified states (for details see [6]).

\section{$31^{++}$and $2^{++}$spectrum}

The model described in the previous section has nine unknown parameters: four $C_{X}^{(\tau)}(X=A$ or $X=B$ ) parameters describing the contact interactions between the $D$ mesons, $d$ parameter describing the coupling of the charmonium to a pair of $D$ mesons, and four masses of the charmonium states. Ideally, these parameters should be fitted to experimental data. Due to the lack of sufficient data to fix all the unknown parameters several simplifications will be made: isospin breaking mass differences will be ignored, and we will concentrate only on the $J^{P C}=1^{++}$and $J^{P C}=2^{++}$sectors. After these simplifications, the only necessary contact interaction term is $C_{0}=C_{A}+C_{B}$, reducing the total number of unknowns to four: $C_{0}, d$, and the masses of the charmonia in the relevant $J^{P C}$ sectors. Furthermore, 
the observed mass of $X(3872)$ will be used as an experimental input, further reducing the number of unknowns by one.

In the $J^{P C}=1^{++}$sector, for the bare mass of the charmonium, the mass predicted by [8] is used. In the $J^{P C}=2^{++}$sector, the $\chi_{c 2}$ meson is observed to have a mass $m_{\chi_{c 2}}=3906 \mathrm{MeV}$. In this sector, this will be used as an experimental input to identify the bare mass of the $2^{++}$charmonium. This leaves a single free parameter in both of the $J^{P C}$ sectors. In this work, this parameter is chosen to be the $d$ parameter, and the behavior of the spectrum is analyzed as this parameter is changed.

In Table 1 , the spectrum of the $J^{P C}=1^{++}$sector is presented. Note that at the mass of the $X(3872)$, the coupling to the charmonium creates an effective attraction proportional to $d^{2}$. Hence, in order the keep the mass of the $X(3872)$ fixed, for sufficiently large values of $d$, the parameter $C_{0}$ should increase to positive values, resulting even in a repulsive four particle contact interaction. As expected, as $d$ increases, the molecular component of the $X(3872)$ meson gets smaller and smaller, and for very large values of $d, X(3872)$ is dominantly a charmonium state.

The other pole, which in the $d=0$ limit is the pure charmonium state, develops a width as the parameter $d$ starts to get nonzero values. As $d$ increases, this pole moves deep into the complex plane, and then goes below threshold. A further increase in the value of $d$ causes this pole to approach the real axis on the second Riemann sheet (SRS). Once this pole reaches the real axis, it collides with its conjugate pole and two poles start to move on the real axis, one moving towards and the other one moving away from the threshold. Since these poles are on the SRS, they will not have any observable consequences if they are deep in the complex plane, or on the real axis, but much below threshold. In Table 1, we only show the pole that might have an observable effect on line shapes, i.e. the pole that moves towards the threshold.

In Table 2, we present the results we obtain in the $J^{P C}=2^{++}$sector. In this sector, the mass of the $\chi_{c 2}$ meson has been fixed on the observed value $m_{\chi_{c 2}}=3927.2 \pm 2.6 \mathrm{MeV}$ [19]. Note that the closest prediction from the constituent quark model calculations for the mass of the $\chi_{c 2}$ state is $m_{\chi_{c 2}}=3949$

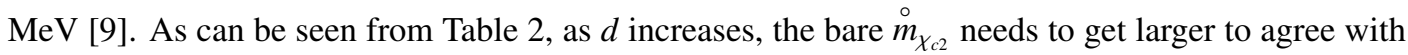
the experiment. If this bare mass is taken to be the prediction of the constituent quark model, it is seen that the discrepancy between constituent quark model predictions, and the bare mass gets smaller. This implies that the origin of the discrepancy between the constituent quark model predictions and the experimental observation for the $\chi_{c 2}$ mass is due to the threshold effects.

The other pole, which corresponds to $X_{2}$, starts as a stable (dominantly) molecular state for small values of the parameter $d$. As $d$ increases, it reaches threshold and crosses to the SRS, and for even larger values of $d$, it moves quickly deep into the complex plane in the SRS, below threshold. Since it is below threshold in the SRS, and deep in the complex plane, it will not have any observable effects, a possible reason for the non observation of this $J^{P C}=2^{++}$partner of $X(3872)$.

\section{Conclusions}

In this talk, the framework, developed in [6], based on heavy quark spin symmetry is presented where both the molecular and the charmonium components of $X(3872)$ can be studied. In the $J^{P C}=1^{++}$and $J^{P C}=2^{++}$sectors, this model has few parameters. The spectrum and properties of the spectrum of this model is studied. It is shown that a large charmonium component can easily be accommodated within this model depending on the parameter $d$. Furthermore, in the $J^{P C}=2^{++}$sector, the origin of the discrepancy between the constituent quark model predictions and the observation of $\chi_{c 2}$ can be identified as the coupling of the charmonium to $D$ mesons, an effect that is ignored in constituent quark models (see [20] for a recent analysis on the effects of meson loops on quark models). 
Table 1. Properties of the $1^{++}$hidden charm poles as a function of $d$. The position of the $X(3872)$ is fixed at $M_{X}=3871.69 \mathrm{MeV}$ in the FRS. The $\chi_{c 1}(2 P)$ pole is located in the SRS. Finally, $d^{\text {crit }}(\Lambda=1 \mathrm{GeV})=0.370 \mathrm{fm}^{1 / 2}$.

\begin{tabular}{c|ccc|cccc}
$\begin{array}{c}d \\
{\left[\mathrm{fm}^{1 / 2}\right]}\end{array}$ & $\begin{array}{c}C_{0 X} \\
{\left[\mathrm{fm}^{2}\right]}\end{array}$ & $\begin{array}{c}g_{D \bar{D}^{*}}^{X(3872)} \\
{\left[\mathrm{GeV}^{-1 / 2}\right]}\end{array}$ & $\tilde{X}_{X(3872)}$ & $\begin{array}{c}\left(m_{\chi_{c 1}}, \Gamma_{\chi_{c 1}}\right) \\
{[\mathrm{MeV}]}\end{array}$ & $\begin{array}{c}g_{D \bar{D}^{*}}^{\chi_{c 1}} \\
{\left[\mathrm{GeV}^{-1 / 2}\right]}\end{array}$ & $\left|\tilde{X}_{\chi_{c 1}}\right|$ & $\tilde{Z}_{\chi_{c 1}}$ \\
\hline 0. & -0.789 & 0.90 & 1 & $(3906.0,0)$ & 0. & 0.0 .16 & 1. \\
0.05 & -0.774 & 0.89 & 0.98 & $(3906.6,1.9)$ & $0.01+0.16 i$ & 0.02 & $0.99+0.01 i$ \\
0.1 & -0.731 & 0.87 & 0.92 & $(3908.2,7.9)$ & $0.03+0.31 i$ & 0.06 & $0.96+0.05 i$ \\
0.15 & -0.659 & 0.83 & 0.84 & $(3910.5,19.2)$ & $0.07+0.44 i$ & 0.14 & $0.92+0.11 i$ \\
0.20 & -0.559 & 0.78 & 0.75 & $(3912.4,37.8)$ & $0.14+0.56 i$ & 0.23 & $0.87+0.19 i$ \\
0.25 & -0.429 & 0.73 & 0.66 & $(3912.0,67.0)$ & $0.24+0.65 i$ & 0.36 & $0.82+0.31 i$ \\
0.30 & -0.271 & 0.68 & 0.57 & $(3903.9,112.8)$ & $0.38+0.73 i$ & 0.55 & $0.77+0.50 i$ \\
0.35 & -0.084 & 0.63 & 0.49 & $(3864.5,185.2)$ & $0.63+0.85 i$ & $>1$ & $0.70+1.01 i$ \\
$d^{\text {crit }}$ & 0.000 & 0.61 & 0.47 & $(3798.3,209.4)$ & $0.93+1.09 i$ & $>1$ & $0.53+2.12 i$ \\
0.375 & 0.020 & 0.61 & 0.46 & $(3754.4,186.4)$ & $1.21+1.37 i$ & $>1$ & $0.29+3.66 i$ \\
0.3775 & 0.031 & 0.61 & 0.46 & $(3701.6,93.5)$ & $2.19+2.39 i$ & $>1$ & $-0.44+12.27 i$ \\
0.40 & 0.132 & 0.59 & 0.43 & $(3827.1,0)$ at SRS & 0.96 & $\tilde{X}_{\chi_{c 1}}<0$ & 2.07 \\
0.45 & 0.376 & 0.55 & 0.37 & $(3850.9,0)$ at SRS & 0.63 & $\tilde{X}_{\chi_{c 1}}<0$ & 1.52 \\
0.5 & 0.649 & 0.51 & 0.32 & $(3858.4,0)$ at SRS & 0.51 & $\tilde{X}_{\chi_{c 1}}<0$ & 1.36 \\
1.0 & 4.963 & 0.29 & 0.11 & $(3869.7,0)$ at SRS & 0.21 & $\tilde{X}_{\chi_{c 1}}<0$ & 1.08 \\
2.0 & 22.217 & 0.15 & 0.03 & $(3871.3,0)$ at SRS & 0.10 & $\tilde{X}_{\chi_{c 1}}<0$ & 1.02 \\
$d \gg d^{\text {crit }}$ & $\frac{d^{2}}{m_{\chi_{c 1}}-M_{X}}$ & $O(1 / d)$ & $O\left(1 / d^{2}\right)$ & $\left(M_{X}-O\left(\frac{1}{d^{2}}\right), 0\right)$ at SRS & $O(1 / d)$ & $\tilde{X}_{\chi_{c 1}}=-O\left(\frac{1}{d^{2}}\right)$ & $1+O\left(\frac{1}{d^{2}}\right)$ \\
\hline
\end{tabular}

Table 2. Properties of the $2^{++}$hidden charm poles as a function of $d$. The position of the dressed $\chi_{c 2}(2 P)$ is fixed at $m_{\chi c 2}^{\text {exp }}=3927.2 \mathrm{MeV}$ in the FRS, and we also give the $X(3872)$ meson-molecular probabilities $\left(\tilde{X}_{X(3872)}\right)$ for each value of $d$.

\begin{tabular}{|c|c|c|c|c|c|c|c|}
\hline $\begin{array}{c}d \\
{\left[\mathrm{fm}^{1 / 2}\right]}\end{array}$ & $\tilde{X}_{X(3872)}$ & $\begin{array}{c}g_{D^{*} \bar{D}^{*}}^{\chi_{c 2}} \\
{\left[\mathrm{GeV}^{-1 / 2}\right]}\end{array}$ & $\tilde{X}_{\chi c 2}$ & $\begin{array}{c}\stackrel{\circ}{m}_{\chi c 2} \\
{[\mathrm{MeV}]}\end{array}$ & $\begin{array}{c}M_{X_{2}}-2 M_{D^{*}}-i \frac{\Gamma_{X_{2}}}{2} \\
{[\mathrm{MeV}]}\end{array}$ & $\begin{array}{c}g_{D^{*} \bar{D}^{*}}^{X_{2}} \\
{\left[\mathrm{GeV}^{-1 / 2}\right]}\end{array}$ & $\tilde{X}_{X_{2}}$ \\
\hline 0. & 1 & 0.0 & 0.0 & 3927.2 & -5.6 & 0.97 & 1. \\
\hline 0.05 & 0.98 & 0.27 & 0.01 & 3927.8 & -4.5 & 0.90 & 0.996 \\
\hline 0.10 & 0.92 & 0.51 & 0.02 & 3929.6 & -1.8 & 0.67 & 0.991 \\
\hline 0.15 & 0.84 & 0.69 & 0.04 & 3932.2 & -0.0 at SRS & $-0.12 i$ & $>1$ \\
\hline 0.20 & 0.75 & 0.82 & 0.05 & 3935.2 & -6.4 at SRS & $-0.76 i$ & $>1$ \\
\hline 0.22 & 0.71 & 0.86 & 0.06 & 3936.4 & -21.2 at SRS & $-1.24 i$ & $>1$ \\
\hline 0.25 & 0.66 & 0.90 & 0.06 & 3938.3 & $-28.3-\frac{72.9}{2} i$ & $0.23-0.65 i$ & $0.47+0.32 i$ \\
\hline 0.30 & 0.57 & 0.95 & 0.07 & 3941.2 & $-31.2-\frac{162.8}{2} i$ & $0.03+0.67 i$ & $0.48-0.04 i$ \\
\hline 0.35 & 0.49 & 0.96 & 0.07 & 3943.8 & $-59.5-\frac{312.6}{2} i$ & $0.30+0.71 i$ & $0.52-0.39 i$ \\
\hline
\end{tabular}

\section{Acknowledgments}

This research has been supported by the Spanish Ministerio de Economía y Competitividad and European FEDER funds under the contracts FIS2014-51948-C2-1-P, FIS2014-57026-REDT and SEV2014-0398, by Generalitat Valenciana under contract PROMETEOII/2014/0068 and by TUBITAK under contract 114F234. 


\section{References}

[1] S. K. Choi et al. (Belle), Phys. Rev. Lett. 91, 262001 (2003).

[2] D. Acosta et al. (CDF Collaboration), Phys. Rev. Lett. 93, 072001 (2004).

[3] V. M. Abazov et al. (D0 Collaboration), Phys. Rev. Lett. 93, 162002 (2004).

[4] B. Aubert et al. (BaBar Collaboration), Phys. Rev. D 71, 071103 (2005).

[5] R. Aaij et al. (LHCb Collaboration), Eur. Phys. J. C 72, 1972 (2012).

[6] E. Cincioglu, J. Nieves, A. Ozpineci and A. U. Yilmazer, arXiv:1606.03239 (2016).

[7] P. G. Ortega, J. Segovia, D. R. Entem, and F. Fernandez, Phys. Rev. D 81, 054023 (2010).

[8] J. Segovia, D. R. Entem, F. Fernandez, and E. Hernandez, Int. J. Mod. Phys. E 22, 1330026 (2013).

[9] D. Ebert, R. N. Faustov, and V. O. Galkin, Eur. Phys. J. C 71, 1825 (2011).

[10] C. Hanhart, Yu. S. Kalashnikova, A. E. Kudryavtsev, and A. V. Nefediev, Phys. Rev. D 85, 011501 (2012).

[11] D. Gamermann, J. Nieves, E. Oset and E. Ruiz Arriola, Phys. Rev. D 81, 014029 (2010).

[12] C. Hidalgo, J. Nieves, A. Ozpineci and V. S. Zamiralov, Int. J. Mod. Phys. Conf. Ser. 26, 1460110 (2014).

[13] BaBar collaboration, B. Aubert et al., Phys. Rev. Lett. 102, 132001 (2009).

[14] R. Aaij et al., LHCb Collaboration, Nucl.Phys. B886, 665 (2014).

[15] M. Takizawa and S. Takeuchi, PTEP 2013, 0903D01 (2013).

[16] J. Nieves and M. P. Valderrama, Phys. Rev. D 86, 056004 (2012).

[17] R. Casalbuoni, A. Deandrea, N. Di Bartolomeo, R. Gatto, F. Feruglio and G. Nardulli, Phys. Lett. B 302, 95 (1993).

[18] P. Colangelo, F. De Fazio and T. N. Pham, Phys. Rev. D 69, 054023 (2004).

[19] K. A. Olive et al. (Particle Data Group), Chin. Phys. C38, 090001 (2014).

[20] I. K. Hammer, C. Hanhart and A. V. Nefediev, arXiv:1607.06971 (2016). 